\title{
Plädoyer für eine Ausbildung in «inklusiver» Medizin ...
}

\section{Patrick Bodenmann}

Prof. Dr. med., Inhaber des Lehrstuhls für Medizin für gefährdete Bevölkerungsgruppen an der Fakultät für Biologie und Medizin der Universität Lausanne, Leiter der medizinischen Poliklinik der Universität Lausanne

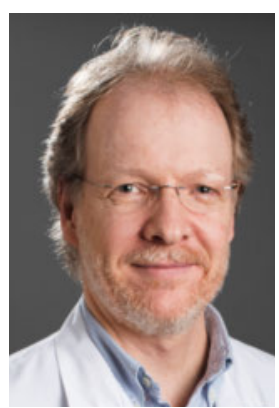

Auf Einladung der Mathematisch-Naturwissenschaftlichen und Medizinischen Fakultät der Universität Freiburg erwarteten mich am Nachmittag des 28. Februar 80 Studierende im 2. Jahr des Bachelor-Studiengangs um 15.15 Uhr für zwei Unterrichtseinheiten zum Thema Zwangsmigration. Ich stelle ihnen meine Themen vor: Epidemiologische Realitäten, nationale und lokale Massnahmen zur Betreuung von Zwangsmigranten, spezielle Pathologien und Schlussfolgerungen über den Nutzen des Erwerbs kulturübergreifender klinischer Kompetenzen, um so eine gleichwertige Pflege für Minderheiten (nicht nur mit Migrationshintergrund) bereitstellen zu können. Um 17.40 Uhr bin ich noch immer im selben Auditorium und die Fragen aufmerksamer Jugendlicher mit mir zugewandten, interessierten Gesichtern finden kein Ende. Ein Gefühl der Zufriedenheit durchströmt mich angesichts der Tatsache, dass eine meiner Hauptaktivitäten im Ausbildungsbereich liegt. Am 21. März erwarten meinen Linguisten-Kollegen und mich um die 100 Studierende im 1. Jahr des Master-Studiengangs der Universität Lausanne zum Thema Umgang mit tauben und schwerhörigen Patientinnen und Patienten. Die 15-minütige Pause reicht nicht aus, um allen Fragen gerecht zu werden - vor allem jenen an unseren Dolmetscher für französische Gebärdensprache, auch in der 45-minütigen Unterrichtseinheit für unsere taube "Patientin», die sich für zwei kurze Szenen vor den Zuhörerinnen und Zuhörern bereitgestellt hatte.

Einen Kommentar im Lancet vom 20. Januar 2018 überschreibt Prof. M. Marmot mit dem Titel «Inclusion health: adressing the causes of the causes" [1]. Anhand des sozialen Gradienten zeigt er auf, inwieweit die Tatsache, dass die Menschen auf unterschiedlichen Stufen der sozialen Leiter stehen, einer echten sozialen Kohäsion zuwiderläuft: Personen ohne festen Wohnsitz, Drogenabhängige, Sexarbeitende, Gefangene ... es gibt viele Menschen, die sozial ausgeschlossen sind und Gefahr laufen, in Sachen Gesundheit ungerecht behandelt zu werden. Auf den Kommentar folgt eine erste Systematisierung der Literatur mit Metaanalyse, aus der hervorgeht, dass die Mortalität dieser vier Gruppen bei Männern um das 8-Fache und bei Frauen um das 12-Fache höher liegt als beim Durchschnitt [2]. In einer zweiten systematischen Review stellen die Autoren fest, dass es bei der Betreuung dieser gefährdeten Gruppen Optimierungspotenzial gibt: Case management, besonderes Augenmerk auf die Wohnbedingungen (Modell Housing first), spezielle, ganz auf die individuellen Bedürfnisse ausgerichtete pharmakologische Behandlung, Integration der sozialen Gefährdung in die Anamnese etc. [3]. Es gibt viele Komponenten für eine Inklusivmedizin mit Blick auf jene, die am stärksten gefährdet sind.

Neben dem Interesse junger künftiger Kolleginnen und Kollegen und den in einer renommierten medizinischen Zeitschrift veröffentlichten Informationen aus fundierten Studien sollte uns auch die soziale und gesundheitliche Realität vor Ort von der Bedeutung einer solchen Ausbildung überzeugen: In der Schweiz gelten $7,5 \%$ der Bevölkerung als armutsgefährdet, 10 bis $15 \%$ der Betroffenen verzichteten in den letzten zwölf Monaten aus Kostengründen auf medizinische Versorgung. Sexuelle Minderheiten zeigen Komorbiditäten im mentalen Bereich und starke Abhängigkeitsprobleme und etwa $13 \%$ sind schwerhörig oder taub. Alle diese Minderheiten (und andere) laufen Gefahr, in der Gesundheitsversorgung ungleich behandelt $\mathrm{zu}$ werden, in einem Land, das deutlich mehr tun kann für mehr Gerechtigkeit in der Pflege [4].

Die jüngste Ausgabe von PROFILES (Principal Relevant Objectives and Framework for Integrated Learning and Education in Switzerland) [5], ein neues Medium zur Festsetzung von Zielvorgaben und Lerninhalten der medizinischen Lehrpläne in der Schweiz, unterstreicht die Bedeutung der Ausbildung in einer sozialen, klinischen Inklusivmedizin, im Rahmen derer die jungen Assistentinnen und Assistenten beispielsweise lernen, ihre Kompetenzen bereits am ersten Praxisalltag im Umgang mit Transgendern, Personen ohne festen Wohnsitz oder Gehörlosen umzusetzen.

Wir haben die Chance - aber auch die Verantwortung unseren Studierenden solche Studiengänge anzubieten So können wir sie begleiten und dazu anregen, die gesamte Bandbreite ihrer künftigen Patientinnen und Patienten im Blick zu haben. Wir können sie für jene Bereiche begeistern, die für die Umsetzung einer inklusiveren - und damit gerechteren - Medizin unabdingbar sind. 